\title{
CoYoT1 Clinic:
}

\section{Innovative Telemedicine Care Model for Young Adults with Type 1 Diabetes}

\author{
Jennifer K. Raymond, MD, MCR, Cari L. Berget, RN, BSN, CDE, Kimberly A. Driscoll, PhD, \\ Kaitlin Ketchum, BS, Cynthia Cain, NP, CDE, and John F. "Fred" Thomas, $\mathrm{PhD}^{2-4}$
}

\begin{abstract}
Background: Young adults with type 1 diabetes (T1D) face many challenges in managing their diabetes, resulting in suboptimal glycemic control and often loss to follow-up. Comprehensive strategies are needed to engage this population in diabetes care and improve outcomes. This pilot study investigated the feasibility and acceptability of the Colorado Young Adults with Type 1 Diabetes (CoYoT1) Clinic - an innovative clinical care model for young adults with T1D, incorporating telemedicine and peer interactions.

Subjects and Methods: Forty-five patients with T1D, 18-25 years of age, participated in this study. Patients completed one routine, diabetes clinic appointment, using Health Insurance Portability and Accountabilityapproved, Web-based videoconferencing from a location of their choosing. The clinic visit consisted of an individual appointment with a diabetes provider and a group appointment with other young adults, facilitated by a certified diabetes educator. Patients completed a satisfaction survey and reported the time lost from school or work to complete the virtual appointment compared with time typically lost to complete a traditional, in-person, visit. Results: Patients reported high levels of satisfaction with the virtual clinic and high levels of perceived support from the peer interaction. Additionally, patients reported saving over $6 \mathrm{~h}$ from their work or school day when completing their diabetes clinic visit virtually instead of in-person.

Conclusions: In this cross-sectional pilot study, the CoYoT1 Clinic, incorporating Web-based videoconferencing and peer interactions, was feasible and acceptable for young adults with T1D. This model may potentially increase engagement with diabetes care in the young adult population. However, further research is needed to fully evaluate the intervention.
\end{abstract}

\section{Introduction}

$\mathbf{F}$ OR Patients with type 1 diabetes (T1D), glycemic control is suboptimal during adolescence and young adulthood (18-25 years old) and, in the last few years, is only becoming more discouraging. ${ }^{1,2}$ Recent data from the T1D Exchange $^{2}$ showed that glycemic control worsened in the 13-25-year-old age group from 2012 to 2014, whereas all other age groups saw either no change or an improvement in glycemic control. Highest hemoglobin A1c (HbA1c) levels occurred a 19 years of age, with an average HbAlc of $9.2 \%$ $(77 \mathrm{mmol} / \mathrm{mol})$, and only $13 \%$ of young adults achieved the recommended $\mathrm{HbA} 1 \mathrm{c}$ target of $<7.0 \%(53 \mathrm{mmol} / \mathrm{mol})$.
Young adults with T1D, especially those leaving home and attending college, face multiple challenges when managing their diabetes that likely impact their glycemic control, such as competing social, academic, and vocational priorities, lack of and change in routines, and reduction in diabetes-specific support due to moving away from parents. ${ }^{3,4}$ Additionally, young adults with diabetes have a high risk of diabetic ketoacidosis (DKA), diabetes complications, and diminishing mental health, ${ }^{1,2,5-8}$ making them a population in need of support.

Complicating matters, patients with T1D in this $18-25$ year-old age group often lose connection with their diabetes provider and experience a significant lapse in recommended

\footnotetext{
${ }^{1}$ Barbara Davis Center for Childhood Diabetes, University of Colorado Anschutz Medical Campus, Aurora, Colorado.

${ }^{2}$ Department of Telehealth, Children's Hospital Colorado, Aurora, Colorado.

${ }^{3}$ Department of Psychiatry, University of Colorado School of Medicine, Aurora, Colorado.

${ }^{4}$ Department of Epidemiology, University of Colorado School of Public Health, Aurora, Colorado.
} 
routine diabetes care. ${ }^{3,4,9-11}$ Young adults in this transitional stage report feeling dissatisfied with the transfer to adult care and believe their specific needs and diabetes management challenges are not sufficiently addressed in pediatric or adult care settings, ${ }^{4,12}$ likely contributing to their disengagement with medical care. Additionally, there is evidence to suggest worsening of glycemic control with the transition to adult care. ${ }^{13}$ Comprehensive interventions are needed, not only to improve the transfer from pediatric to adult care, but, more importantly, to keep young adults engaged in clinical care, address their diabetes needs, and improve glycemic control.

Maximizing technological resources to allow for more frequent contact with medical providers has resulted in improved medical outcomes in various populations. Telemedicine technology has been used to reach patients struggling to attend routine medical care appointments, ${ }^{14}$ including pediatric patients with T1D. ${ }^{15}$ Use of telemedicine to manage T1D in adolescents has been well accepted, resulting in improved access to care, self-management, and glycemic control. ${ }^{14,15}$

Additionally, alternative medical care approaches, specifically group visits, also known as shared medical appointments, have successfully increased patient and provider satisfaction, strengthened follow-up rates, and improved outcomes in multiple patient populations. ${ }^{16-18}$ Furthermore, increasing peer support for young adults with T1D has been suggested as an avenue to improve mental health and adherence with diabetes self-care, ${ }^{3,4,19}$ and group visits are an efficient way to incorporate peer support into routine medical care. "Team Clinic," a group clinical care model ${ }^{20}$ piloted for adolescents with T1D, was found to be an efficient way to provide developmentally tailored clinical care with the added benefit of peer support. As many as six adolescents with T1D participated in a group session, facilitated by a certified diabetes educator, in order to discuss diabetes management challenges with other adolescents, in addition to seeing their diabetes medical provider. Participants in Team Clinic reported high levels of satisfaction with their clinic appointments.

Given the potential for telemedicine to increase access to medical care and group appointments to increase patient satisfaction and provide peer support, an alternative approach to clinical care for young adults with T1D that incorporates both innovative techniques is desirable. The Colorado Young Adults with T1D (CoYoT1) (pronounced "coyote") Clinic was designed to meet the medical care needs of young adults with T1D in a technology-driven, developmentally tailored, group visit model. The purpose of this pilot study was to investigate the feasibility and acceptability of the CoYoT1 Clinic intervention, an innovative approach to providing medical care to young adults with T1D.

\section{Subjects and Methods \\ Overall CoYoT1 Clinic structure}

The CoYoT1 Clinic structure was based on the Team Clinic model ${ }^{20}$ and adapted to a virtual setting. For each patient, the CoYoT1 Clinic consisted of an individual appointment with a diabetes provider (MD or nurse practitioner) and a group appointment, with other young adults, facilitated by a certified diabetes educator. Four patients were scheduled for each group appointment. In order to streamline the two components of the clinic, two of the four patients completed the provider visit prior to the group appointment, and the remaining two patients completed the provider visit after the group appointment. All four patients participated in the group session together. For each patient, the entire CoYoT1 Clinic visit lasted 50-60 min: $20-30 \mathrm{~min}$ for the individual appointment with a diabetes provider and $30 \mathrm{~min}$ for the group session (Fig. 1).

Participants completed the virtual clinic visit from home, or another location of their choosing, using a Web-based, videoconferencing software, Vidyo ${ }^{\mathrm{TM}}$ (Vidyo, Inc., Hackensack, NJ). This platform delivers end-to-end encryption, provides Health Insurance Portability and Accountability-compliant security, and easily integrates into existing healthcare environments. Prior to the scheduled clinic visit, participants uploaded their diabetes management devices (insulin pump, glucometer, and/or continuous glucose monitor) to a Webbased program, allowing the diabetes provider to access the patients' device reports via the Internet. Additionally, patients completed HbA1c measurements, and any other needed laboratory measurements, from an outside laboratory (e.g., primary care provider's office, student health center, or private lab company). The lab results were faxed from the testing laboratory to the provider at the diabetes center. The only requirement for participation in the CoYoT1 Clinic was access to the Internet via a device (e.g., tablet, laptop, personal computer, smartphone) equipped with a webcam and speakers. Participants joined the clinic through a link they received via e-mail.

\section{CoYoT1 Clinic group appointment}

The group session began with an icebreaker activity and then moved to a patient-driven, facilitator-mediated discussion on a topic relevant to young adults with T1D. Topics included the following: (1) stress management; (2) building a diabetes support system, including how to advocate for

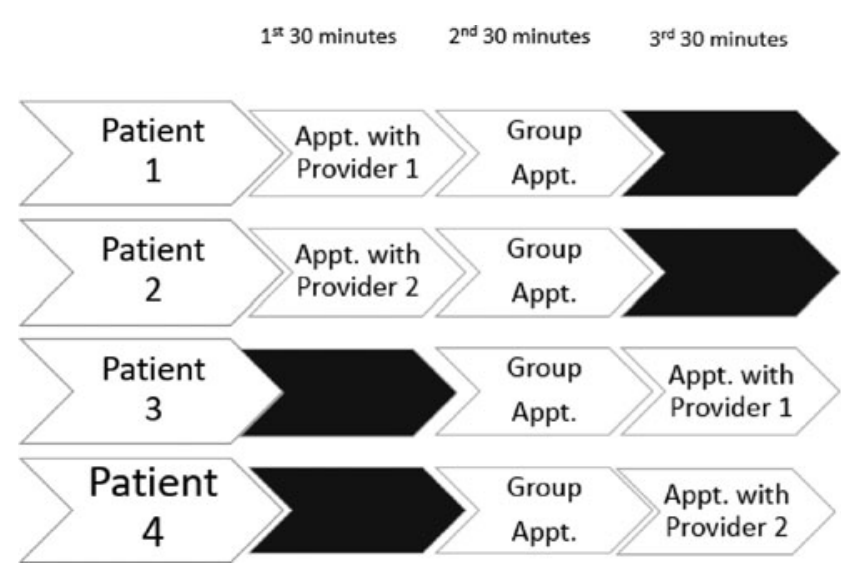

FIG. 1. Colorado Young Adults with Type 1 Diabetes (CoYoT1) Clinic structure. Two diabetes providers and one certified diabetes educator staffed each CoYoT1 Clinic session. For each patient, the CoYoT1 Clinic, including a group and an individual appointment (appt.), lasted 5060 min. Patients complete their individual appointment either prior to or after the group appointment. 
oneself in a college or work setting; (3) practical aspects of day-to-day diabetes management (e.g., prescriptions, problem solving, emergencies); (4) high-risk activities, such as alcohol or drug use; (5) use of insulin pumps and continuous glucose monitors; (6) diabetes burnout; and (7) participants' "diabetes story." Topics were chosen based on patient interest, and not all topics were discussed in each group. The facilitator guided the session by using open-ended questions to elicit discussion as well as active listening techniques to steer the discussion and encourage interaction among participants.

\section{CoYoT1 Clinic individual appointment with diabetes provider}

During the individual appointment with the diabetes provider, the provider reviewed lab results and glycemic information obtained from the patient's device downloads with the patient, discussed diabetes management and barriers to optimal glycemic control, and provided individualized recommendations. Additionally, the patient had the opportunity to discuss any concerns with the provider during this time. At the end of the visit, the provider summarized the plan of care and recommendations and then e-mailed a copy to the patient. Of note, if patients had additional questions to be addressed by ancillary staff (e.g., dietary guidance for high cholesterol), staff members contacted patients by e-mail or phone to review questions or concerns.

\section{Frequency of clinic visits in the preceding year}

Chart review was conducted to determine the number of diabetes clinic visits completed by each patient in the 15 months prior to enrollment in the CoYoT1 Clinic. This was used to determine a baseline measure of clinical care engagement and adherence with the American Diabetes Association recommendation of quarterly clinic visits (every 3 months) for young adults with T1D. ${ }^{11}$

\section{Time savings}

In order to determine if the CoYoT1 Clinic resulted in time savings from work or school, patients reported the amount of time lost from work or school to complete the CoYoT1 Clinic appointment as well as the time previously lost from work or school to attend a traditional, in-person diabetes appointment. A paired $t$ test was computed to determine the time savings from the CoYoT1 Clinic.

\section{Patient satisfaction survey}

In order to assess patient satisfaction with the CoYoT1 Clinic, patients completed a satisfaction survey online after completing their group and individual appointment. The satisfaction survey consisted of 12 statements for which participants rated their level of agreement or disagreement on a 5-point Likert scale ranging from $1=$ strongly disagree to $5=$ strongly agree. Nine statements assessed satisfaction with the use of Web-based videoconference technology, and the remaining three statements assessed satisfaction with the group appointment. A total satisfaction score was calculated by summing the survey responses, with a maximum score of 60 reflecting high satisfaction. Additionally, two subscales were calculated: a technology satisfaction subscale and a group satisfaction subscale. The maximum technology subscale score was 45 , and the maximum group subscale score was 15 .

\section{Participants}

Patients between 18 and 25 years of age, currently being seen in a pediatric diabetes center, were invited to participate in the CoYoT1 Clinic. Non-English-speaking patients and patients with severe behavioral or psychological disorders, making participation in a group difficult, were excluded. Newly diagnosed patients who had not completed the diabetes center's standard new onset diabetes education were also excluded. In total, 71 young adults were contacted; 17 declined participation, and 54 completed informed consent to participate in the CoYoT1 Clinic, resulting in a $76 \%$ recruitment rate. Of those who consented to participate in the intervention, seven withdrew from the study before scheduling a CoYoT1 Clinic appointment, and two patients did not show up for their scheduled appointment, resulting in 45 participants. Of the seven patients who withdrew prior to beginning the study, five patients withdrew because of scheduling conflicts, one had recently transferred care to an adult endocrinologist, and one patient decided against participation in the group appointment. Institutional Review Board review and approval were completed prior to intervention implementation. Informed consent was completed virtually, using the videoconference platform used for the intervention. After informed consent was completed, research staff provided instructions to participants about how to complete their laboratory measurements and upload their diabetes devices from home.

\section{Results}

Baseline characteristics of the sample are given in Table 1. Forty-one patients $(91 \%)$ completed both the individual provider appointment and the group appointment of the CoYoT1 clinic, whereas four patients $(9 \%)$ completed only the individual

Table 1. Baseline Characteristics of Colorado Young Adults with Type 1 Diabetes Clinic Participants

\begin{tabular}{lc}
\hline Characteristic & Value \\
\hline Hemoglobin A1c & $8.7 \% \pm 1.6 \%$ \\
& $(71.6 \mathrm{mmol} / \mathrm{mol})$ \\
Gender (female) & $55.8 \%$ \\
Age (years) & $19.8 \pm 1.6$ \\
Completed diabetes clinic visits in the last & $2.5 \pm 1.2$ \\
$\quad$ year $(n)$ & $8.6 \pm 4.6$ \\
Diabetes duration (years) & $2.93 \pm 2.1$ \\
Blood glucose readings per day $(n)$ & $76 \%$ \\
Use insulin pumps & $34 \%$ \\
Use multiple daily injections & $5 \%$ \\
Use continuous glucose monitors & $29 \%(n=13)$ \\
Last appointment $>5$ months prior to & \\
$\quad$ CoYoT1 visit & $22 \%(n=10)$ \\
$\geq 4$ or more diabetes clinic visits in the & \\
preceding 15 months & \\
\hline
\end{tabular}

Data are mean \pm SD values or percentages as indicated.

CoYoT1, Colorado Young Adults with Type 1 Diabetes. 
provider appointment. Of the 41 patients who completed both components of the clinic appointment, four patients completed the provider appointment and the group appointment on different days. For two patients, this was due to technical problems during the group appointment, resulting in the group session being canceled and the patients participating in a group appointment on a different date. Fortunately the problems encountered during these occurrences were remedied, and additional technical challenges were not encountered during the pilot. For the other two patients, the original group appointment was canceled because of scheduling difficulties, where an insufficient number of patients was scheduled for the group, and those patients completed their individual provider appointment as originally scheduled and joined a group appointment on a different date. Prior to the CoYoT1 Clinic visit, 38 patients (84\%) successfully downloaded their diabetes devices to a Web-based program, and 41 patients $(91 \%)$ completed laboratory measurements.

\section{Frequency of clinic visits in the preceding year}

Prior to completing the virtual CoYoT1 Clinic visit, patients completed an average of 2.5 diabetes clinic visits in the preceding year. Only 10 patients $(22 \%)$ completed the recommended four visits in the preceding year, eight patients (18\%) completed just one visit, and two patients (4\%) were not seen at all in the previous year. At the time of enrollment in the CoYoT1 Clinic, 13 patients $(29 \%)$ had not been seen in a clinic in at least 5 months, but once recruited to the CoYoT1 Clinic, they were seen within 2-4 weeks.

\section{Time savings from the CoYoT1 Clinic}

Patients reported an average round trip travel time of $131 \mathrm{~min}$ to the diabetes center. Additionally, $66 \%$ of patients reported they typically lost time from work or school when completing a traditional diabetes clinic visit at the diabetes center. Patients reported losing an average of $6.05 \mathrm{~h}$ for a traditional, in-person, diabetes clinic visit. Comparatively, when patients completed their clinic visit virtually, via the CoYoT1 Clinic, patients reported losing an average of $0.08 \mathrm{~h}$, with only $2 \%$ of patients reporting any lost time. This resulted in a time savings of $5.97 \mathrm{~h}$ for patients completing their diabetes clinic visit via the CoYoT1 Clinic compared with the traditional, in-person diabetes clinic visit $(P<0.0001)$ (Table 2).

Table 2. Participants' Report of Time Savings With THE Colorado Young Adults WITH Type 1 Diabetes Clinic

\begin{tabular}{lc}
\hline Average time & Value \\
\hline Travel for in-person diabetes clinic visit $(n=32)$ & $131 \mathrm{~min}$ \\
Lost from work/school day for CoYoT1 Clinic & $0.08 \mathrm{~h}$ \\
$\quad \begin{array}{l}\text { appointment }(n=31) \\
\text { Lost from work/school day for traditional, }\end{array}$ & $6.05 \mathrm{~h}$ \\
$\quad$ in-person clinic visit $(n=31)$ & $5.97 \mathrm{~h}^{\mathrm{a}}$ \\
Savings for online appointment & \\
\hline${ }^{\text {a } P<0.0001 \text { for difference. }} \begin{array}{l}\text { CoYoT1, Colorado Young Adults with Type 1 Diabetes. }\end{array}$
\end{tabular}

\section{Satisfaction with the CoYoT1 Clinic}

Patients reported high levels of satisfaction with the CoYoT1 Clinic overall. The mean total satisfaction score was $51.7 \pm 5.6$ (maximum score $=60$ ). The mean technology satisfaction subscale score was $39.5 \pm 3.9$ (maximum score $=$ 45 ), and the mean group satisfaction subscale score was $12.2 \pm 2.3$ (maximum score $=15$ ). Patients felt comfortable using the videoconference technology and desired to have another online appointment. Patients reported that the online visit was easier to complete and took less time to complete than a traditional, in-person clinic visit. Additionally, the majority of patients enjoyed talking to other young adults with T1D during the group appointment and reported a high level of perceived support from the interaction (Table 3). Interestingly, patients connected with one another following the visits, but this was not fostered or encouraged by the medical team. Young adults initiated this contact independently.

The two providers (one pediatric endocrinologist and one nurse practitioner) participating in the CoYoT1 Clinic were highly satisfied with the experience. Both providers found it offered them the opportunity to connect with a traditionally "unreachable" and "disengaged" population. The pediatric endocrinologist (J.K.R.) found the online appointments resulted in a more authentic partnership with her patients, and patients seemed more engaged in their care. Also of note, patients involved in the CoYoT1 Clinic did not necessarily know the provider they were seeing online, but patients appeared quite comfortable with interacting with a new provider in the CoYoT1 Clinic.

\section{Discussion}

To our knowledge, this is the first study examining Webbased videoconferencing for routine diabetes care in this population with patients at home, or another location of their choosing. The CoYoT1 Clinic, a telemedicine clinic using a group visit care model, was designed to address several of the barriers to engaging young adults in care by making clinical care easily accessible, reducing time lost from work or school to complete clinic appointments, and increasing peer support. The findings of this pilot study suggest that the CoYoT1 Clinic is feasible and acceptable for young adults with T1D. High levels of satisfaction, a $100 \%$ completion rate of the provider visit and a $91 \%$ completion rate of the group appointment, and success in obtaining clinical data from patients suggest that the CoYoT1 Clinic is potentially a better clinical care model for the young adult population with T1D. The majority of patients were successful in downloading their diabetes devices at home, a task they were unfamiliar with prior to beginning the study, and nearly all patients completed laboratory measurements prior to their appointment. As a result, providers were able to access the same information during the virtual CoYoT1 Clinic visit as during a traditional, in-person clinic appointment.

Research shows that competing life priorities, such as school, work, and social demands, are barriers to care for young adults with T1D. ${ }^{4,9}$ The patients in our study reported saving over $6 \mathrm{~h}$ from their work or school day by completing their diabetes clinic visit virtually, which may improve adherence with routine diabetes care appointments. Offering the opportunity to interact with healthcare providers via 
Table 3. Satisfaction with the Colorado Young Adults with Type 1 Diabetes Clinic $(N=38)$

\begin{tabular}{lccc}
\hline Statement & $\begin{array}{c}\text { Agree or } \\
\text { strongly agree }\end{array}$ & $\begin{array}{c}\text { Disagree or } \\
\text { Neutral } \\
\text { strongly disagree }\end{array}$ \\
\hline I felt comfortable using technology for my online visit. & $95 \%$ & $5 \%$ & $0 \%$ \\
I felt as though the online visit respected my privacy. & $92 \%$ & $5 \%$ & $3 \%$ \\
I got everything I needed during the online appointment. & $90 \%$ & $8 \%$ & $2 \%$ \\
The online appointment was much easier than my normal appointments. & $76 \%$ & $24 \%$ & $0 \%$ \\
Compared to a normal appointment, the online appointment took a lot more & $11 \%$ & $11 \%$ & $78 \%$ \\
$\quad$ time. & $92 \%$ & $8 \%$ & $0 \%$ \\
I would recommend this technology to others. & $95 \%$ & $5 \%$ & $0 \%$ \\
I would like to have an online appointment again. & $70 \%$ & $27 \%$ & $3 \%$ \\
I liked talking to other young adults during the online visit. & $90 \%$ & $10 \%$ & $0 \%$ \\
I felt supported during the group visit. & $97 \%$ & $0 \%$ & $3 \%$ \\
I felt comfortable asking my doctor questions through the online visit. & $92 \%$ & $8 \%$ & $0 \%$ \\
I was able to speak freely and express myself. & $70 \%$ & $19 \%$ & $11 \%$ \\
I liked the group style of the appointment. & &
\end{tabular}

technology, from a patient-chosen location, may increase access to diabetes care by making it easier for young adult patients to fit diabetes clinic visits into their busy schedules, despite competing priorities, and overcome other barriers to care, such as travel time.

Professionally led support groups have shown potential to improve diabetes self-care behaviors and reduce diabetes distress for young adult patients in their 20s. ${ }^{19}$ Additionally, research has found young adults value peer-led education and shared experiences, and diabetes education may be enhanced by incorporating peer-led diabetes education with clinicianled diabetes education. ${ }^{12}$ Seventy percent of the patients who participated in the CoYoT1 Clinic enjoyed the opportunity to discuss diabetes challenges with their peers, and $20 \%$ reported feeling neutral. However, $90 \%$ felt supported by the interaction. Patients currently reporting ambivalence to the group format may, over time, become more comfortable interacting in the group setting and benefit further from peer interaction. A group visit structure is an efficient way to incorporate diabetes self-management education and peer support into routine diabetes care, without requiring additional efforts on the part of the patient or healthcare professionals. Incorporating peer support into the clinic visit may hold potential to enhance mental health, ensure effective diabetes education, and improve problem solving related to common barriers to optimal diabetes self-management for this vulnerable population. Additionally, addressing this need through a home telemedicine intervention makes the peer interaction easily accessible to patients and more efficient than interventions requiring additional visits or even inperson shared medical appointments.

\section{Limitations and future directions}

The results of this study should be interpreted in the context of a few limitations. The participants were not randomly assigned to participate in the CoyoT1 Clinic, and we did not include a control group for comparison. It is possible the patients in our study were more motivated to pursue clinical care than the greater 18-25-year-old population with T1D. However, in the real world, patients are not randomly assigned to clinical models and will choose medical care environments that they prefer. It was not difficult to find participants for this study, with a $76 \%$ recruitment rate, indicating the likelihood of a high level of interest in using technology for health care in this population. Furthermore, the participants exhibited a history of less than recommended annual visit frequency, with only $22 \%$ of the participants completing the recommended four annual visits in the preceding year. This demonstrates a high level of acceptability of this care model, even among a cohort with a history of suboptimal visit frequency.

Because of the lack of a control group and the relatively small patient sample completing only one clinic visit, we cannot determine if the CoYoT1 Clinic resulted in increased access to diabetes care or improved clinical outcomes for young adults with T1D. An additional limitation is incomplete satisfaction survey completion by the patient population, with only 38 out of 45 participants $(84 \%)$ completing the survey. However, the purpose of this pilot study was to assess feasibility and acceptability of the CoYoT1 Clinic. The nearuniversal patient satisfaction is impressive, despite these limitations, and suggests potential that warrants further investigation into the CoYoT1 Clinic model of care. We are in the process of conducting a larger trial that includes a control group in order to investigate the impact of the CoYoT1 Clinic on access to diabetes care, adherence to self-care recommendations, and improvements in glycemic control over a 12-month time period. Furthermore, gathering data on the provider's experience will be important for future evaluation of the CoYoT1 Clinic care model.

In addition to the clinical and research implications of the CoYoT1 Clinic, this study has policy implications as well. Telemedicine is growing as a healthcare delivery model, and many states have insurance laws requiring equal reimbursement for clinical services delivered in-person or via telemedicine. ${ }^{21}$ However, many insurers require that patients be located at a "qualifying originating site" (i.e., hospital, medical clinic, etc.) in order for telemedicine services to be covered, posing challenges for reimbursement of clinical services delivered virtually where patients are located at home or other location and not at a medical facility. However, a new insurance law in New York, taking effect in January 2016, does not include the requirement of an originating site for commercial insurance coverage of telemedicine services, ${ }^{22}$ indicating potential for telemedicine reimbursement no matter where the 
patient is located. Although this is a positive first step, how this law will play out is uncertain at this time. Additional research, including a cost-effectiveness analysis and, ultimately, a randomized controlled trial, is needed to advocate for policy change ensuring telemedicine clinical interventions, with patients located at home or other location of their choosing, would be a reimbursable option for young adult patients.

\section{Acknowledgments}

The authors would like to thank and acknowledge funding for the study by The Leona M. and Harry B. Helmsley Charitable Trust. The authors would also like to thank the young adults who participated in the research.

\section{Author Disclosure Statement}

No competing financial interests exist.

\section{References}

1. Wood JR, Miller KM, Maahs DM, et al.: Most youth with type 1 diabetes in the T1D Exchange Clinic Registry do not meet American Diabetes Association or International Society for Pediatric and Adolescent Diabetes clinical guidelines. Diabetes Care 2013;36:2035-2037.

2. Miller KM, Foster NC, Beck RW, et al.: Current state of type 1 diabetes treatment in the U.S.: updated data from the T1D Exchange clinic registry. Diabetes Care 2015;38:971978.

3. Edwards D, Noyes J, Lowes L, et al.: An ongoing struggle: a mixed-method systematic review of interventions, barriers and facilitators to achieving optimal self-care by children and young people with type 1 diabetes in educational settings. BMC Pediatr 2014;14:228.

4. Garvey KC, Beste MG, Luff D, et al.: Experiences of health care transition voiced by young adults with type 1 diabetes: a qualitative study. Adolesc Health Med Ther 2014;5:191198.

5. Johnson B, Elliott J, Scott A, et al.: Medical and psychological outcomes for young adults with Type 1 diabetes: no improvement despite recent advances in diabetes care. Diabet Med 2014;31:227-231.

6. Colton PA, Olmsted MP, Daneman D, et al.: Eating disorders in girls and women with Type 1 diabetes: a longitudinal study of prevalence, onset, remission, and recurrence. Diabetes Care 2015;38:1212-1217.

7. Butwicka A, Frisen L, Almquist C, et al.: Risks of psychiatric disorders and suicide attempts in children and adolescents with type 1 diabetes: a population-based cohort study. Diabetes Care 2015;38:453-459.

8. Bryden KS, Dunger DB, Mayou RA, et al.: Poor prognosis of young adults with type 1 diabetes: a longitudinal study. Diabetes Care 2003;26:1052-1057.

9. Duke DC, Raymond JK, Shimomaeda L, et al.: Recommendations for transition from pediatric to adult diabetes care: patients' perspectives. Diabetes Manage 2013;3: 297-304.

10. Garvey KC, Wolpert HA, Laffel LM, et al.: Health care transition in young adults with type 1 diabetes: barriers to timely establishment of adult diabetes care. Endocr Pract 2013;19:946-952.

11. Peters A, Laffel L; American Diabetes Association Transitions Working Group: Diabetes care for emerging adults: recom- mendations for transition from pediatric to adult diabetes care systems: a position statement of the American Diabetes Association, with representation by the American College of Osteopathic Family Physicians, the American Academy of Pediatrics, the American Association of Clinical Endocrinologists, the American Osteopathic Association, the Centers for Disease Control and Prevention, Children with Diabetes, The Endocrine Society, the International Society for Pediatric and Adolescent Diabetes, Juvenile Diabetes Research Foundation International, the National Diabetes Education Program, and the Pediatric Endocrine Society (formerly Lawson Wilkins Pediatric Endocrine Society). Diabetes Care 2011;34:24772478.

12. Wiley J, Westbrook M, Long J, et al.: Diabetes education: the experiences of young adults with type 1 diabetes. Diabetes Ther 2014;5:299-321.

13. Lotstein DS, Seid M, Klingensmith G, et al.: Transition from pediatric to adult care for youth diagnosed with type 1 diabetes in adolescence. Pediatrics 2013;131:e1062-e1070.

14. Guljas R, Ahmed A, Chang K, et al.: Impact of telemedicine in managing type 1 diabetes among school-age children and adolescents: an integrative review. J Pediatr Nurs 2014;29:198-204.

15. Wood CL, Clements SA, McFann K, et al.: Use of telemedicine to improve adherence to American Diabetes Association standards in pediatric type 1 diabetes. Diabetes Technol Ther 2016;18:7-14.

16. Sanchez I: Implementation of a diabetes self-management education program in primary care for adults using shared medical appointments. Diabetes Educ 2011;37:381-391.

17. Rising SS: Centering pregnancy. An interdisciplinary model of empowerment. J Nurse Midwifery 1998;43: 46-54.

18. Heyworth L, Rosenblum R, Burgess JF Jr: Influence of shared medical appointments on patient satisfaction: a retrospective 3-year study. Ann Fam Med 2014;12:324330.

19. Markowitz JT, Lafell LM: Transitions in care: support group for young adults with Type 1 diabetes. Diabet Med 2012;29:522-525.

20. Raymond JK, Shea JJ, Berget C, et al.: A novel approach to adolescents with type 1 diabetes: the team clinic model. Diabetes Spectr 2015;28:68-71.

21. Lacktman NM: Five Telemedicine Trends Transforming Health Care in 2016. November 17, 2015. https://www .healthcarelawtoday.com/2015/11/17/five-telemedicinetrends-transforming-health-care-in-2016/ (accessed March 9, 2016).

22. Lacktman NM, Rosen EJ: Changes to New York Telehealth Coverage Coming Soon. October 19, 2015. https:// www.healthcarelawtoday.com/2015/10/19/changes-to-newyork-telehealth-coverage-coming-soon/ (accessed March 9, 2016).

Address correspondence to: Jennifer K. Raymond, $M D, M C R$ Barbara Davis Center for Childhood Diabetes University of Colorado Anschutz Medical Campus Mail Stop A140 1775 Aurora Court Aurora, CO 80045

E-mail: Jennifer.raymond@ucdenver.edu 